\title{
Gravitational force and fallacy of space deformation
}

\author{
Enrique Martinez Viladesau \\ Escuela Técnica Superior de Ingenieros Industriales de Barcelona (ETSIUB), Spain \\ E-mail addresses: emviladesau@gmail.com \\ Received Jan 2019 \\ Received in revised: Feb 2020 \\ Published: March 2020
}

\begin{abstract}
In the present paper, we attempt to explain what we really do not know or understand. For this, we assume to accept approaches that go against reason and common sense. We will try to give a view, about inaccuracy arguments state that the gravity force associated with mass, is caused by the deformation of the space adjacent to it. To move from the real world to the world of fantasy, we only needed to support the misconception of the time dilation in the fallacy of the space deformation. We will use this paper to expose some writings that contribute to masking logical reasoning.
\end{abstract}

Keywords: Gravitational force, Space deformation

C2020 The Authors. Published by Fundamental Journals. This is an open access article under the CC BY-NC https://creativecommons.org/licenses/by-nc/4.0/

https://doi.org/10.14331/ijfps.2020.330134

\section{INTRODUCTION}

Using a logical reasoning to be able to consider as a fallacy the idea of the space deformation. We will analyze the variables that intervene in the physical phenomenon of the attraction forces between the masses. For this, using gravitational Newton's law, $F=G M m / d^{2}$. Here $F$, is the force between two masses $M$ and $m$ separated by a distance $d$ and, $G$ is the universal gravitation constant. Newton's law expresses that a force appears among the masses, but does not tell why it is generated. Here it is the mental fantasies appear to try to explain what is unknown. The investigation is not to go against common sense but we suggest performing the following analysis. a) A physical phenomenon is produced between two known objects involved in the phenomenon. We call them masses $M 1$ and $M 2$. b) This physical phenomenon is produced and sustained on a platform outside the variables of the phenomenon. We identify it as space. c) There must be a connection enter and the two objects, the masses, to make that interaction between them. This link is the variable that we do not know and that we intend to identify. Perhaps there are neutrinos that moving around the outer space and through all the masses establish the connection between two masses. Here we leave for consideration and analysis. The above three points can help us to define the following thoughts.

We separate the actors that intervene in the physical phenomenon, from the support or platform on which it is performed. Using the metaphor, we will say that, the scenario in which the function is developed is space and the actors are masses and the dialogue between them is the link between them. 


\section{AGAINST A DISCOVERY OF THE CAUSES OF THE PHENOMENON, AN EXPLANATION IS GIVEN AGAINST THE COMMON SENSE}

We see that Einstein explain the existence of the gravitational force due to a curved space. The presence of the Earth deforms the space that causes another mass to approach and fall on it. Here the question may is that: Can we materialize the space and make the bodies navigate with it? How should we interpret the case in which, for example, a mass is so far from Earth that the force of gravity does not act? Should we interpret that in this case the space has been unfolding or flattening? We think of the familiar idea that says, Space is what remains of an empty room when its walls collapse. We should not give him material attributes.

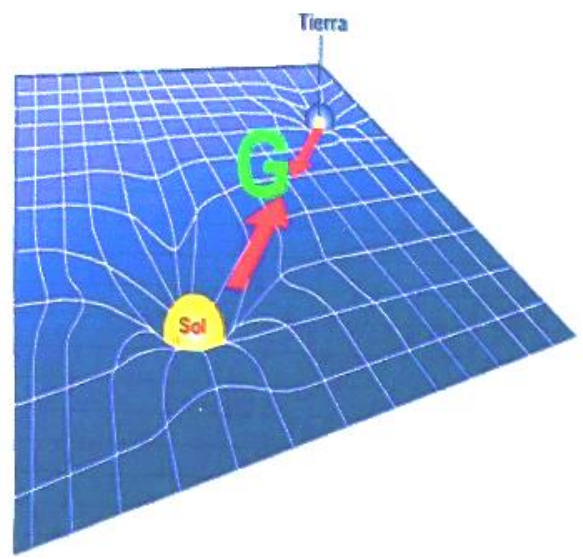

Fig 1. Aims to illustrate such an idea

\section{MENTAL EXPERIMENTS}

Thinking that, as far as the human mind can come and science will not have to leave logical reasoning to try to show what we do not understand. We designs two mental experiments in order to invalidate the idea that space intervenes as a variable to consider in the generation of gravitational forces. These approaches will be identified as, 1- Experiment without the intervention of space and 2- Experiment with equal spaces between the acting masses.

\section{FIRST MENTAL EXPERIMENT: WITHOUT INTERVENTION OF SPACE}

We remember that we separate two different entities, the space and the masses that occupy this space. In this approach if the appearance of attraction forces between two masses be $F a$, and we omit the intervention of space, then we wonder if these forces will really appear. If with the above-mentioned omission, we be able to observe that those forces continue to exist; this will allow us to affirm that the concept of space and its deformation are not causative agents of the physical phenomenon of which we are dealing.

Suppose two masses located in the sidereal space, touching each other, or very close to each other, in which we can see that there is practically no space between them. Fig 2 , then this question comes, Can we say that there is no attraction force between them because there is no space?

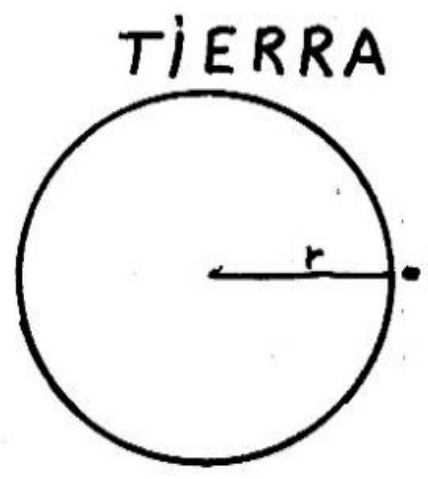

Fig 2. Tries to reveal this circumstance.

Without worrying about the scale of values of its dimensions, we have drawn a large mass that may correspond to the globe and, practically above it, another small mass, which could be for example a test particle with mass $m_{p}$.

The reality confirms that we cannot say that since there is no space between them that can be deformed, no there is gravity. If we try to lift the $m_{p}$ then we will realize that there will be appear an attraction force. Doubt may arise when asked if, in the absence of space, how the masses interact. In the previous paragraph when talking about the possible existence of subatomic particles, we have commented that, it seems that what produces this connection is present both in the space (support of the physical phenomenon that we are considering) and inside the acting masses themselves. Perhaps here it involved the exchange of own particles corresponding subatomic each of the two masses? We note here as information that in the mental experiment, we are considering and applying Newton's formula the value of the variable: Distance $d$ would be the value of the radius $r$ of the Earth. Later, in the next paragraph, we will see this criterion is in order to obtain an acceleration value of a mass is equal 9.8 $\left(\mathrm{m} / \mathrm{sec}^{2}\right)$

\section{SECOND MENTAL EXPERIMENT: WITH EQUALITY OF SPACES BETWEEN THE ACTING MASSES}

In this experiment, we consider two different masses $m_{1}$ and $m_{2}$, which are locating in the same distances $d_{1}=d_{1}$ from a larger mass $M_{T}$ as shown in Fig 3 .

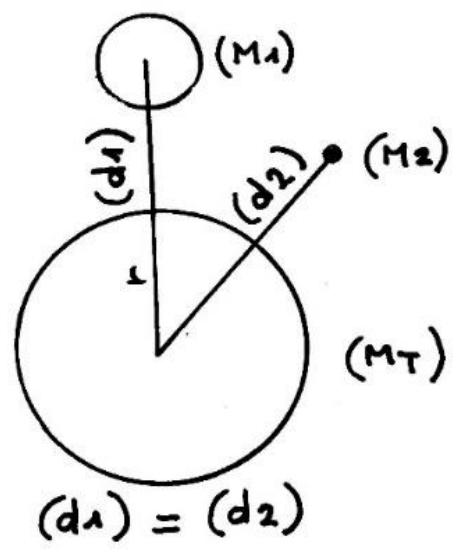

Fig 3 
Suppose the big ball is, Earth with mass is $M_{T}$. Let us see how, we would reason if we were willing to accept that space is deformed. Our reasoning is as follow. If we consider no space as the support medium where the physical phenomenon develops, if not as a variable of the Phenomenon, we should admit that, at equal distances to the mass $M_{T}$, that is, $d_{1}=d_{1}$, the unfolding of space should be the same and the same answers would be obtained. The attraction forces $F_{a}$ should be the same however, this does not happen and we have $F_{a 1} \neq$ $F_{a 2}$. Taking our reasoning to the absurd, we can say that space treat as something material by considering the same piece of this something and should be obtain the same results whereas it does not happen. For example, two different masses, with $m_{1}=1 / \mathrm{kg}$ and $m_{1}=62.5 \times 10^{3} / \mathrm{kg}$ fall from the same height towards the surface of the Earth. Therefore, the attraction forces can be as $F=9.834 N$ and $F=61.464 \times$ $10^{4} N$. Since the two masses fall is 20 meters, but then this distance is negligible compared to the radius of the Earth, it is neglect able in the calculations. What we are now interested in distinguishing is the difference between these two attraction forces that are located in the same distance from a third mass, and this is not consistent with the fallacy of the deformation of space. What, should be clear is that the acceleration of both two masses are same as $\left(61,464 \times 10^{4}\right) /\left(62,5 \times 10^{3}\right)=$ $9,834 \mathrm{~m} / \mathrm{sec}^{2} \quad$ and 9,834/1 $=9,834 \mathrm{~m} / \mathrm{sec}^{2}$ (Martínez, 2015). Regarding these results when calculating the forces, we must clarify the following points. We must bear in mind that what we are investigating is the concept force and not the concept of acceleration.

\section{REFERENCES}

Martínez, E. (2015). Teoría de la relatividad. Una falsa teoría: Una falsa teoría (Spanish Edition): Punto Rojo.

Viladesau, E. M. (2019). Theory of Relativity: The Fallacy of the Principle of Equivalence. International Journal of

\section{CONCLUSION}

We investigate the cause and not the effects. It is interesting to consider this conclusion since in some books this distinction is not clear. The experiment discussed here, as in the first example, we could also assume that the two test masses are touching the surface of the Earth. In this circumstance, their distances would be equal to the sum of the radii of the two spheres. Perhaps, the confusion in which the well-known physicist submits us, with the idea that space bends, we wonder if the interior of the two spheres must be considered as a space. We must be clear about the distinction between space and bodies. The attractive mass must be considered as a whole. As a body whose center of gravity is precisely at the center of this body. Here we note that the value obtained from 9.834 $\mathrm{m} / \mathrm{sec}^{2}$, is approximately equal to the value and it is not accident. The reader may find its origin in the experiment Cavendish. We notify that we have highlighted the word "approximately" whose conclusion can be drawn from the final formula and that this value is only obtained when the attractor mass is Earth. In trying to inform ourselves about the subject of the gravitational forces, we have stumbled upon some writings that, trying to justify the unjustifiable, mix erroneous concepts. Any of them citing the Theory of Relativity. For this reason, we dedicate ourselves in this paragraph to try to fade some doubts and to clarify ideas. To comment on these questions we have chosen the information that appears on (Viladesau, 2019), corresponding to the topic we are dealing with.

Fundamental Physical Sciences (IJFPS), 9(1), 6-9. https://doi.org/10.14331/ijfps.2019.330122 